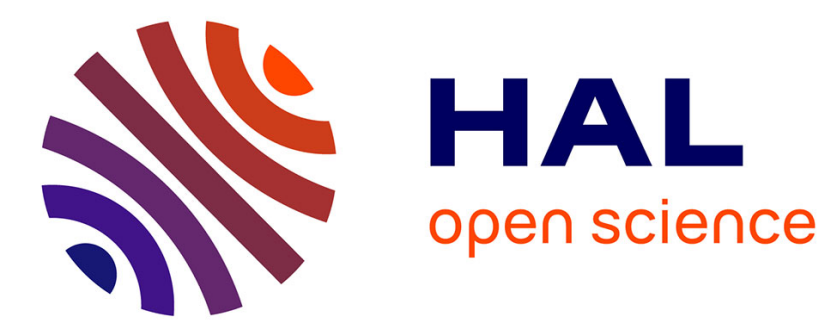

\title{
Long time bounds for the periodic Benjamin-Ono-BBM equation
}

\author{
Youcef Mammeri
}

\section{To cite this version:}

Youcef Mammeri. Long time bounds for the periodic Benjamin-Ono-BBM equation. Nonlinear Analysis: Theory, Methods and Applications, 2009, 71 (10), pp.5010 - 5021. 10.1016/j.na.2009.03.078 . hal-01090385

\section{HAL Id: hal-01090385 \\ https://hal.science/hal-01090385}

Submitted on 3 Dec 2014

HAL is a multi-disciplinary open access archive for the deposit and dissemination of scientific research documents, whether they are published or not. The documents may come from teaching and research institutions in France or abroad, or from public or private research centers.
L'archive ouverte pluridisciplinaire HAL, est destinée au dépôt et à la diffusion de documents scientifiques de niveau recherche, publiés ou non, émanant des établissements d'enseignement et de recherche français ou étrangers, des laboratoires publics ou privés. 


\title{
Long time bounds for the periodic Benjamin-Ono-BBM equation
}

\author{
Y. Mammeri \\ Laboratoire Paul Painlevé, CNRS UMR 8524, Université des Sciences et Technologies de Lille, \\ 59655 Villeneuve d'Ascq Cedex, France. \\ Email: youcef.mammeri@math.univ-lille1.fr
}

March 2009

\begin{abstract}
We consider the periodic Benjamin-Ono equation, regularized in the same manner the Benjamin-BonaMahony equation is found from the Korteweg-de Vries one [3], namely the equation $u_{t}+u_{x}+\alpha u u_{x}+\beta H\left(u_{x t}\right)=0$, where $H$ is the Hilbert transform, $\alpha$ the quotient between the characteristic waves amplitude and the depth of the waves and $\beta$ the quotient between this depth and the wavelength. We show that the solution, starting from an initial datum of size $\varepsilon$, remains smaller than $\varepsilon$ for a time scale of order $\left(\varepsilon^{-1} \frac{\beta}{\alpha}\right)^{2}$, whereas the local well-posedness gives only a time of order $\varepsilon^{-1} \frac{\beta}{\alpha}$.
\end{abstract}

Keywords. BO-BBM equation, KP equations, local and global well-posedness, long time bounds

MS Codes. 35B10, 35B60, 35Q53, 76B03, 76B55

\section{Introduction}

The small amplitude long waves moving inside a nonhomogeneous fluid (of infinite depth) are modelled in 1 -dimension by the Benjamin-Ono equation $[2,7,13,12]$

$$
u_{t}+u_{x}+\alpha u u_{x}-\beta H\left(u_{x x}\right)=0,
$$

where $H$ denotes the Hilbert transform in the $x$-direction, $\alpha$ the quotient between the characteristic waves amplitude and the depth of the waves and $\beta$ the quotient between this depth and the wavelength. We recall $0<\alpha, \beta \leq 1$. Since this equation is obtained correcting, at the second order, the transport equation $u_{t}+u_{x}=0$, we deduce the BO-BBM equation [3]

$$
u_{t}+u_{x}+\alpha u u_{x}+\beta H\left(u_{x t}\right)=0 .
$$

First of all, we prove the global in time well-posedness of the Cauchy problem associated with the BOBBM equation (0.1) using the Brezis-Gallouët's method.

\section{Theorem 0.1}

Let $0<\alpha, \beta \leq 1$ and $s>1 / 2$. Let $f \in H_{0}^{s}(\mathbb{T})$. Then there exists a unique global in time solution $u \in \mathcal{C}\left(\mathbb{R}, H_{0}^{s}(\mathbb{T})\right)$ of the Cauchy problem associated with (0.1) of initial datum $f$.

In particular, we deduce that the norm $\|u(t)\|_{1 / 2}$ is conserved by BO-BBM flow, i.e. for all $t \in \mathbb{R}$, $\|u(t)\|_{1 / 2}=\|f\|_{1 / 2}$. We are then wondering how does the other norms $H_{0}^{s}(\mathbb{T})$, for $s>1 / 2$, behave? As 
a consequence of the local in time well-posedness of the Cauchy problem associated with the BO-BBM equation, for $0<\alpha, \beta \leq 1$ and $s>1 / 2$, there exist two constants $C_{1}>0$ and $C_{2}>0$ such that, if $\|f\|_{s} \leq \varepsilon$, then for $|t| \leq C_{2} \varepsilon^{-1} \frac{\beta}{\alpha}$, the solution $u$ satisfies

$$
\|u(t)\|_{s} \leq C_{1} \varepsilon .
$$

The goal of this work is to prove that the bound (0.2) can be true more long time. Our result reads as follows.

\section{Theorem 0.2}

Let $0<\alpha, \beta \leq 1$ and $s>1 / 2$. There exist $0<\varepsilon_{0}<\frac{\beta^{2}}{\alpha}, C_{1}>0$ and $C_{2}>0$ such that if $f \in H_{0}^{s}(\mathbb{T})$ with $\|f\|_{s} \leq \varepsilon$, for $\left.\varepsilon \in\right] 0, \varepsilon_{0}[$, then the unique solution $u$ of the periodic BO-BBM equation (0.1) with initial datum $f$, satisfies for $|t| \leq C_{2}\left(\varepsilon^{-1} \frac{\beta}{\alpha}\right)^{2}$,

$$
\|u(t)\|_{s} \leq C_{1} \varepsilon .
$$

The principle of the proof consists in rewriting the BO-BBM equation in the normal form

$$
v_{t}+L(v)=F(v),
$$

where $L$ is the operator given by the linear BO-BBM evolution and $F$ a multilinear operator of order strictly higher than 2 . The idea is to ameliorate the quadratic nature of the nonlinearity of the BO-BBM equation. In our work, we are inspired by the method introduced by Tzvetkov [15], and $F$ will be a trilinear operator. In a more general way, this method is called Poincaré's theory of normal forms [1].

The constraint of smallness on $\varepsilon_{0}$ does not depend on $\alpha$ and $\beta$ when $\frac{\beta^{2}}{\alpha} \geq 1$. We also notice that the fact that $\varepsilon_{0}$ is chosen in a such way $\varepsilon<\frac{\beta^{2}}{\alpha}$, with $0<\varepsilon<\varepsilon_{0}$, implies that the extended time $\left(\varepsilon^{-1} \frac{\beta}{\alpha}\right)^{2}$ is higher than the one found by the local existence $\varepsilon^{-1} \frac{\beta}{\alpha}$.

Let us fix $\alpha=1$. When $\beta$ tends to zero, the persistence time of the bound $(0.3)$ given by the theorem 0.2 also tends to zero. That is not surprising because the formal limit, when $\beta$ tends to zero, of the $\mathrm{BO}-\mathrm{BBM}$ equation is the Burgers one

$$
u_{t}+u_{x}+u u_{x}=0 .
$$

The existence time of the Cauchy problem associated with this equation, for an initial datum of size $\varepsilon$, is of order $\varepsilon^{-1}$ and this time is maximum.

We mention that the model describes, in concrete terms, the small amplitude long waves moving at the level of a thermocline. For example, in the strait of Gibraltar, the studied waves propagate at $200 \mathrm{~m}$ deep inside the Mediterranean sea (of total depth $900 \mathrm{~m}$ ), have an amplitude between $50 \mathrm{~m}$ and $80 \mathrm{~m}$, for a wavelength from $1 \mathrm{~km}$ to $2 \mathrm{~km}$ [10], in particular $\frac{1}{4}=\frac{50}{200} \leq \alpha \leq \frac{80}{200}=\frac{2}{5}$ and $\frac{1}{10}=\frac{200}{2000} \leq \beta \leq \frac{200}{1000}=\frac{1}{5}$.

We will use the following notations : $\mathbb{T}=\mathbb{R} /(2 \pi \mathbb{Z})$ is the one-dimensional torus, $\mathbb{Z}^{*}$ the set of nonzero integers. For $s \in \mathbb{R}$, we denote $H_{0}^{s}(\mathbb{T})$ the space of zero $x$-mean value functions equipped with the norm

$$
\|u(t)\|_{s}=\left(\sum_{k \in \mathbb{Z}^{*}}|k|^{2 s}|\hat{u}(k)|^{2}\right)^{1 / 2},
$$


where $\hat{u}$ denotes the Fourier transform defined by

$$
\hat{u}(k)=\int_{\mathbb{T}} \mathrm{e}^{-i k x} u(x) d \mu(x) .
$$

The measure $d \mu(x)$ is chosen proportional to the Lebesgue one on $\mathbb{T}$ and normalized such that

$$
u(x)=\sum_{k \in \mathbb{Z}^{*}} \mathrm{e}^{i k x} \hat{u}(k) .
$$

The Hilbert transform $H$ in the $x$-direction is defined in such a way that $\widehat{H(u)}(k):=-\mathrm{i} \operatorname{sgn}(k) \widehat{u}(k)$.

The paper is subdivided into three sections. In Section 1, we prove the existence and uniqueness of solution for the BO-BBM equation. Section 2 deals with the main result, namely the proof of the theorem 0.2. In Section 3, the result found by Tzvetkov [15], concerning the KP equation (2d-model of long dispersive nonlinear waves propagation) is extended and we explain why the method no longer apply to its regularized version.

\section{Existence and Uniqueness of solution}

We consider the Cauchy problem, for $t \in \mathbb{R}$, and $x \in \mathbb{T}$,

$$
\begin{aligned}
u_{t}+u_{x}+\alpha u u_{x}+\beta H\left(u_{x t}\right) & =0 \\
u(x, 0) & =f(x) .
\end{aligned}
$$

The equation (1.1) is rewritten as

$$
u_{t}+\frac{\partial_{x}}{1+\beta\left|\partial_{x}\right|} u=-\frac{\alpha}{2} \frac{\partial_{x}}{1+\beta\left|\partial_{x}\right|} u^{2}
$$

where $\frac{\partial_{x}}{1+\beta\left|\partial_{x}\right|}$ is defined by the Fourier symbol $\sigma(k):=\frac{i k}{1+\beta|k|}$.

The existence and uniqueness of local in time solution are first of all proved.

\section{Theorem 1.1}

Let $0<\alpha, \beta \leq 1$ and $s>1 / 2$. Let $f \in H_{0}^{s}(\mathbb{T})$. There exists a constant $C_{0}>0$, depending only on $s$, such that for

$$
T=\frac{1}{C_{0}\|f\|_{s}} \frac{\beta}{\alpha},
$$

there exists a unique solution $u \in \mathcal{C}\left([-T, T] ; H_{0}^{s}(\mathbb{T})\right)$ of the periodic Cauchy problem (1.1)-(1.2).

Moreover, for all $M>0$ with $\|f\|_{s} \leq M$ and $\|g\|_{s} \leq M$, there exists $C_{1}>0$ such that the solutions $u$ and $v$, with $f$ and $g$ as initial data respectively, satisfy for $t \in[-T, T]$, with $T=\frac{1}{C_{0} M} \frac{\beta}{\alpha}$,

$$
\|u(t)-v(t)\|_{s} \leq C_{1}\|f-g\|_{s} .
$$

Proof. The proof is done for positive time.

It is enough to remark for $u \in H_{0}^{s}(\mathbb{T})$

$$
\left\|\frac{\partial_{x}}{1+\beta\left|\partial_{x}\right|} u\right\|_{s}^{2}=\sum_{k \in \mathbb{Z}^{*}}|k|^{2 s}\left(\frac{k}{1+\beta|k|}\right)^{2}|\hat{u}(k)|^{2},
$$


and since $1+\beta|k| \geq \beta|k|$, for $k \in \mathbb{Z}^{*}$, we deduce

$$
\alpha\left\|\frac{\partial_{x}}{1+\beta\left|\partial_{x}\right|} u\right\|_{s} \leq \frac{\alpha}{\beta}\|u\|_{s}
$$

Let $T>0$. The Duhamel formula implies that $u$ is the solution of the Cauchy problem (1.1)-(1.2) if and only if $u$ is the solution of the following equation, for $t \in[0, T]$,

$$
u(t)=\Phi u(t):=S_{t} f-\frac{\alpha}{2} \int_{0}^{t} S_{t-\tau}\left(\frac{\partial_{x}}{1+\beta\left|\partial_{x}\right|} u^{2}\right)(\tau) d \tau,
$$

with $S_{t} f:=\sum_{k \in \mathbb{Z}^{*}} \mathrm{e}^{i k x} \mathrm{e}^{-i t \frac{k}{1+\beta|k|}} \hat{f}(k)$. We aim to apply the fixed point theorem. We deduce from the preceding formula and the inequality (1.3) that, for $t \in[0, T]$,

$$
\|\Phi u(t)\|_{s} \leq\|f\|_{s}+\frac{\alpha}{2} \int_{0}^{t}\left\|\frac{\partial_{x}}{1+\beta\left|\partial_{x}\right|} u^{2}\right\|_{s}(\tau) d \tau,
$$

and since $s>1 / 2$, the Sobolev injection implies that there exists a constant $C_{s}>0$, depending only on $s$, such that

$$
\begin{aligned}
\|\Phi u(t)\|_{s} & \leq\|f\|_{s}+\frac{\alpha}{\beta} C_{s} \int_{0}^{t}\|u\|_{s}^{2}(\tau) d \tau \\
& \leq\|f\|_{s}+\frac{\alpha}{\beta} C_{s} T\left(\sup _{t \in[0, T]}\|u(t)\|_{s}\right)^{2} .
\end{aligned}
$$

Then there exists $C_{0}>0$ such that for $T=\frac{1}{C_{0}\|f\|_{s}} \frac{\beta}{\alpha}$, the closed ball

$$
\bar{B}_{T}:=\left\{u \in \mathcal{C}\left([0, T] ; H_{0}^{s}(\mathbb{T})\right) ; \sup _{t \in[0, T]}\|u(t)\|_{s} \leq 2\|f\|_{s}\right\}
$$

verifies $\Phi\left(\bar{B}_{T}\right) \subset \bar{B}_{T}$. Indeed, let $u \in \bar{B}_{T}$, the inequality (1.5) becomes

$$
\|\Phi u(t)\|_{s} \leq\|f\|_{s}+\frac{4 C_{s}\|f\|_{s}}{C_{0}}
$$

and $\|f\|_{s}+\frac{4 C_{s}\|f\|_{s}}{C_{0}} \leq 2\|f\|_{s}$ if $C_{0} \geq 4 C_{s}$.

Let $u$ and $v$ in $\bar{B}_{T}$. The Duhamel formula (1.4) gives, for $t \in[0, T]$,

$$
\|\Phi u(t)-\Phi v(t)\|_{s} \leq \frac{\alpha}{2} \int_{0}^{t}\left\|\frac{\partial_{x}}{1+\beta\left|\partial_{x}\right|}\left(u^{2}-v^{2}\right)\right\|_{s}(\tau) d \tau
$$

and the Sobolev injection implies

$$
\begin{aligned}
\|\Phi u(t)-\Phi v(t)\|_{s} & \leq \frac{4 \alpha}{\beta} C_{s}\|f\|_{s} T \sup _{t \in[-T, T]}\|u-v\|_{s}(t) \\
& =\frac{4 C_{s}}{C_{0}} \sup _{t \in[0, T]}\|u-v\|_{s}(t) .
\end{aligned}
$$


For $C_{0}>4 C_{s}$, the map $\Phi$ is a contraction on $\bar{B}_{T}$. Finally, according to the fixed point theorem, there exists a unique solution $u$ of $\Phi u(t)=u(t)$ in $\bar{B}_{T}$.

It remains to prove the continuity with the initial datum. Let $u$ and $v$ solutions of the Cauchy problem (1.1)-(1.2) with initial data $f$ and $g$ respectively, such that $\|f\|_{s} \leq M$ and $\|g\|_{s} \leq M$. The Duhamel formula (1.4) gives for $t \in[0, T]$, with $T=\frac{1}{C_{0} M} \frac{\beta}{\alpha}$,

$$
\begin{aligned}
\|u(t)-v(t)\|_{s} & \leq\|f-g\|_{s}+\frac{\alpha}{2} \int_{0}^{t}\left\|\frac{\partial_{x}}{1+\beta\left|\partial_{x}\right|}\left(u^{2}-v^{2}\right)\right\|_{s}(\tau) d \tau \\
& \leq\|f-g\|_{s}+\frac{2 C_{s}}{C_{0} M}\left(\|f\|_{s}+\|g\|_{s}\right) \sup _{t \in[0, T]}\|u-v\|_{s}(t) .
\end{aligned}
$$

Since $C_{0}>4 C_{s}$, we find

$$
\|u(t)-v(t)\|_{s} \leq\|f-g\|_{s}+\frac{1}{2} \sup _{t \in[0, T]}\|u-v\|_{s}(t)
$$

thus

$$
\sup _{t \in[0, T]}\|u-v\|_{s} \leq 2\|f-g\|_{s}
$$

\section{Remark 1}

The time of the local well-posedness could be higher, especially in the case where $0<\beta \leq \alpha \leq 1$, but we have to impose more regularity for the initial datum [8]. More precisely, let us suppose $s>3 / 2$ and $f \in H_{0}^{s}(\mathbb{T})$. Then, there exists $C_{0}>0$, depending only on $s$, such that for

$$
T=\frac{1}{C_{0}\|f\|_{s}} \frac{1}{\alpha}
$$

there exists a unique solution $u \in \mathcal{C}\left([-T, T] ; H_{0}^{s}(\mathbb{T})\right)$ of the periodic Cauchy problem (1.1)-(1.2).

On the other hand, the choice of $\varepsilon_{0}$ in the theorem 0.2 also implies that the extended time $\left(\varepsilon^{-1} \frac{\beta}{\alpha}\right)^{2}$ is higher than $\varepsilon^{-1} \frac{1}{\alpha}$, the one found by this local existence result.

Now, we prove the global well-posedness of the BO-BBM equation.

\section{Proposition 1}

Let $s>1 / 2$ and $f \in H_{0}^{s}(\mathbb{T})$. Then the solution $u$ of the Cauchy problem (1.1)-(1.2) verifies for all $t \in[-T, T]$

$$
\|u(t)\|_{1 / 2}=\|f\|_{1 / 2} .
$$

Proof. We multiply the equation (1.1) by $u$ and integrate over the $x$-direction, according standard regularization arguments.

To prove the global well-posedness, the Brezis-Gallouët's method [5] is used.

\section{Lemma 1.2}

Let $s>1 / 2$ and $u \in \mathcal{C}\left([-T, T] ; H_{0}^{s}(\mathbb{T})\right)$ the solution of the Cauchy problem (1.1)-(1.2). Then there exists $C_{1}>0$ such that

$$
\|u\|_{L^{\infty}} \leq C_{1}\left(1+\sqrt{\log \left(1+\|u\|_{s}\right)}\right) .
$$


Proof. Let $R>0$, where $R$ is to be chosen. We write

$$
\begin{aligned}
\|u\|_{L^{\infty}} \leq\|\hat{u}\|_{L^{1}} & =\sum_{k \in \mathbb{Z}^{*}}|\hat{u}(k)|=\sum_{|k|<R}|\hat{u}(k)|+\sum_{|k| \geq R}|\hat{u}(k)| \\
& =\sum_{0<|k|<R}\left(\frac{|k|}{|k|}\right)^{1 / 2}|\hat{u}(k)|+\sum_{|k| \geq R}\left(\frac{|k|}{|k|}\right)^{s}|\hat{u}(k)| .
\end{aligned}
$$

The Cauchy-Schwarz inequality gives

$$
\|u\|_{L^{\infty}} \leq\left(\sum_{0<|k|<R}|k||\hat{u}(k)|^{2}\right)^{1 / 2}\left(\sum_{0<|k|<R} \frac{1}{|k|}\right)^{1 / 2}+\left(\sum_{|k| \geq R}|k|^{2 s}|\hat{u}(k)|^{2}\right)^{1 / 2}\left(\sum_{|k| \geq R} \frac{1}{|k|^{2 s}}\right)^{1 / 2} .
$$

Since $s>1 / 2$, there exists $\varepsilon>0$ such that $2(s-\varepsilon)>1$. We deduce

$$
\begin{aligned}
\|u\|_{L^{\infty}} & \leq C\|u\|_{1 / 2} \sqrt{\log (R)}+\frac{\|u\|_{s}}{R^{\varepsilon}}\left(\sum_{|k| \geq R} \frac{1}{|k|^{2(s-\varepsilon)}}\right)^{1 / 2} \\
& \leq C\|u\|_{1 / 2} \sqrt{\log (R)}+C \frac{\|u\|_{s}}{R^{\varepsilon}}
\end{aligned}
$$

We choose $R=\left(1+\|u\|_{s}\right)^{1 / \varepsilon}$ to get

$$
\|u\|_{L^{\infty}} \leq C \varepsilon^{-1 / 2}\|u\|_{1 / 2} \sqrt{\log \left(1+\|u\|_{s}\right)}+C \frac{\|u\|_{s}}{1+\|u\|_{s}} .
$$

\section{Theorem 1.3}

Let $0<\alpha, \beta \leq 1$ and $s>1 / 2$. Let $f \in H_{0}^{s}(\mathbb{T})$. Then there exists a unique global in time solution $u \in \mathcal{C}\left(\mathbb{R}, H_{0}^{s}(\mathbb{T})\right)$ of the Cauchy problem (1.1)-(1.2).

Proof. The time of the local existence given by the theorem 1.1 is denoted $T$ again. For $t \in[-T, T]$, the Duhamel formula is written

$$
u(t)=S_{t} f-\frac{\alpha}{2} \int_{0}^{t} S_{t-\tau}\left(\frac{\partial_{x}}{1+\beta\left|\partial_{x}\right|} u^{2}\right)(\tau) d \tau
$$

with $S_{t} f:=\sum_{k \in \mathbb{Z}^{*}} \mathrm{e}^{i k x} \mathrm{e}^{-i t \frac{k}{1+\beta|k|}} \hat{f}(k)$. We get then

$$
\|u(t)\|_{s} \leq\|f\|_{s}+\frac{\alpha}{2 \beta} \int_{0}^{t}\|u(\tau)\|_{L^{\infty}}\|u(\tau)\|_{s} d \tau .
$$

The preceding lemma 1.2 implies that there exists $C_{1}>0$ such that

$$
\|u(t)\|_{s} \leq\|f\|_{s}+\frac{\alpha}{2 \beta} C_{1} \int_{0}^{t}\left(1+\sqrt{\log \left(1+\|u(\tau)\|_{s}\right)}\right)\|u(\tau)\|_{s} d \tau=: \Psi(t) .
$$

We notice

$$
\begin{aligned}
\Psi^{\prime}(t) & =\frac{\alpha}{2 \beta} C_{1}\left(1+\sqrt{\log \left(1+\|u(t)\|_{s}\right)}\right)\|u(t)\|_{s} \\
& \leq \frac{\alpha}{2 \beta} C_{1}(1+\sqrt{\log (1+\Psi(t)}) \Psi(t) \leq \frac{\alpha}{2 \beta} C_{1}(1+\log (1+\Psi(t)) \Psi(t)
\end{aligned}
$$


in another way, there exists $C_{0}>0$ such that

$$
\frac{d}{d t} \log \left(1+\log (1+\Psi(t)) \leq C_{0}\right.
$$

We deduce that there exist $C_{2}>0$ and $C_{3}>0$ such that for all $t \in[-T, T],\|u(t)\|_{s} \leq \mathrm{e}^{c_{2}} \mathrm{e}^{c_{3} t}$. In particular, $\|u(t)\|_{s}$ remains bounded on every finite time interval and the solution can be extended in time at all $\mathbb{R}$.

\section{Long time bounds for the periodic BO-BBM equation}

\section{Corollary 1}

Let $0<\alpha, \beta \leq 1, s>1 / 2$. Let $\varepsilon>0$ and $f \in H_{0}^{s}(\mathbb{T})$ with $\|f\|_{s} \leq \varepsilon$. Then there exist $C_{0}>0$ and $C_{1}>0$ such that the solution $u$ of the Cauchy problem (1.1)-(1.2) verifies for $|t| \leq C_{0} \varepsilon^{-1} \frac{\beta}{\alpha}$,

$$
\|u(t)\|_{s} \leq C_{1} \varepsilon .
$$

We ask if the solution remains small more long time. To respond, we use a method introduced by Tzvetkov [15].

We first prove some useful results. We recall that, for $k \in \mathbb{Z}, \sigma(k)=\frac{i k}{1+\beta|k|}$.

\section{Lemma 2.1}

The Fourier symbol $\sigma$ satisfies for all $k$ and $k_{1}$ in $\mathbb{Z}^{*}$

$$
\left|\sigma\left(k_{1}\right)+\sigma\left(k-k_{1}\right)-\sigma(k)\right| \geq \beta^{2} \frac{\left|k k_{1}\left(k-k_{1}\right)\right|}{(1+\beta|k|)\left(1+\beta\left|k_{1}\right|\right)\left(1+\beta\left|k-k_{1}\right|\right)} .
$$

Proof. Six cases have to be studied.

i. If $k>0, k_{1}>0$ and $k-k_{1}>0$, we have

$$
\left|\sigma\left(k_{1}\right)+\sigma\left(k-k_{1}\right)-\sigma(k)\right|=\frac{\left|\beta(2+\beta k) k_{1}\left(k-k_{1}\right)\right|}{(1+\beta|k|)\left(1+\beta\left|k_{1}\right|\right)\left(1+\beta\left|k-k_{1}\right|\right)} .
$$

ii. If $k>0, k_{1}>0$ and $k-k_{1}<0$,

$$
\left|\sigma\left(k_{1}\right)+\sigma\left(k-k_{1}\right)-\sigma(k)\right|=\frac{\left|\beta k\left(2+\beta k_{1}\right)\left(k-k_{1}\right)\right|}{(1+\beta|k|)\left(1+\beta\left|k_{1}\right|\right)\left(1+\beta\left|k-k_{1}\right|\right)} .
$$

iii. If $k>0$ and $k_{1}<0$,

$$
\left|\sigma\left(k_{1}\right)+\sigma\left(k-k_{1}\right)-\sigma(k)\right|=\frac{\left|\beta k k_{1}\left(2+\beta\left(k-k_{1}\right)\right)\right|}{(1+\beta|k|)\left(1+\beta\left|k_{1}\right|\right)\left(1+\beta\left|k-k_{1}\right|\right)} .
$$

iv. If $k<0$ and $k_{1}>0$,

$$
\begin{aligned}
\left|\sigma\left(k_{1}\right)+\sigma\left(k-k_{1}\right)-\sigma(k)\right| & =\frac{\left|-\beta k k_{1}\left(2-\beta\left(k-k_{1}\right)\right)\right|}{(1+\beta|k|)\left(1+\beta\left|k_{1}\right|\right)\left(1+\beta\left|k-k_{1}\right|\right)} \\
& =\frac{\beta|k| k_{1}\left(2+\beta\left|k-k_{1}\right|\right)}{(1+\beta|k|)\left(1+\beta\left|k_{1}\right|\right)\left(1+\beta\left|k-k_{1}\right|\right)} .
\end{aligned}
$$


v. If $k<0, k_{1}<0$ and $k-k_{1}>0$,

$$
\begin{aligned}
\left|\sigma\left(k_{1}\right)+\sigma\left(k-k_{1}\right)-\sigma(k)\right| & =\frac{\left|-\beta k\left(2-\beta k_{1}\right)\left(k-k_{1}\right)\right|}{(1+\beta|k|)\left(1+\beta\left|k_{1}\right|\right)\left(1+\beta\left|k-k_{1}\right|\right)} \\
& =\frac{\beta|k|\left(2+\beta\left|k_{1}\right|\right)\left(k-k_{1}\right)}{(1+\beta|k|)\left(1+\beta\left|k_{1}\right|\right)\left(1+\beta\left|k-k_{1}\right|\right)} .
\end{aligned}
$$

vi. If $k<0, k_{1}<0$ and $k-k_{1}<0$,

$$
\begin{aligned}
\left|\sigma\left(k_{1}\right)+\sigma\left(k-k_{1}\right)-\sigma(k)\right| & =\frac{\left|(2-\beta k)\left(-\beta k_{1}\right)\left(k-k_{1}\right)\right|}{(1+\beta|k|)\left(1+\beta\left|k_{1}\right|\right)\left(1+\beta\left|k-k_{1}\right|\right)} \\
& =\frac{(2+\beta|k|) \beta\left|k_{1}\right|\left(k-k_{1}\right)}{(1+\beta|k|)\left(1+\beta\left|k_{1}\right|\right)\left(1+\beta\left|k-k_{1}\right|\right)}
\end{aligned}
$$

Let $D:=\left\{\left(k, k_{1}\right) \in \mathbb{Z}^{2} ; k \neq 0, k_{1} \neq 0, k \neq k_{1}\right\}$ and we define the bilinear operator

$$
B(u, v):=-\frac{\alpha}{2} \sum_{D} \mathrm{e}^{i k x} \frac{i k}{1+\beta|k|} \frac{\hat{u}\left(k_{1}\right) \hat{v}\left(k-k_{1}\right)}{\sigma\left(k_{1}\right)+\sigma\left(k-k_{1}\right)-\sigma(k)} .
$$

\section{Lemma 2.2}

Let $s>1 / 2$. There exists a constant $C>0$ such that for all $u$ and $v$ in $H_{0}^{s}(\mathbb{T})$

$$
\|B(u, v)\|_{s} \leq C \frac{\alpha}{\beta^{2}}\|u\|_{s}\|v\|_{s} .
$$

Proof. By duality, to prove the lemma is equivalent to prove for all $w \in \mathcal{C}_{\text {per }}^{\infty}(\mathbb{T})$

$$
\left|\sum_{D} \widehat{B(u, v)}(k) \hat{w}(k)\right| \leq C \frac{\alpha}{\beta^{2}}\left(\|u\|_{s}\|v\|_{s}\right)\|w\|_{-s} .
$$

Indeed, we have

$$
\|B(u, v)\|_{s}^{2}=\sum_{k \in \mathbb{Z}^{*}}|k|^{2 s}|\widehat{B(u, v)}(k)|^{2}=\sum_{k \in \mathbb{Z}^{*}} \widehat{B(u, v)}(k)\left(|k|^{2 s} \widehat{B(u, v)}(k)\right) .
$$

We set then $\hat{w}(k)=|k|^{2 s} \widehat{B(u, v)}(k)$. We write

$$
\|B(u, v)\|_{s}^{2}=\sum_{k \in \mathbb{Z}^{*}} \widehat{B(u, v)}(k) \hat{w}(k),
$$

and according to the inequality (2.1)

$$
\|B(u, v)\|_{s}^{2} \leq C \frac{\alpha}{\beta^{2}}\left(\|u\|_{s}\|v\|_{s}\right)\|w\|_{-s} .
$$

However

$$
\begin{aligned}
\|w\|_{-s} & =\left(\sum_{k \in \mathbb{Z}^{*}}|k|^{-2 s}|k|^{4 s}|\widehat{B(u, v)}(k)|^{2}\right)^{1 / 2} \\
& =\left(\sum_{k \in \mathbb{Z}^{*}}|k|^{2 s}|\widehat{B(u, v)}(k)|^{2}\right)^{1 / 2}=\|B(u, v)\|_{s} .
\end{aligned}
$$


We define then

$$
\hat{u}_{1}(k)=|k|^{s} \hat{u}(k), \hat{v}_{1}(k)=|k|^{s} \hat{v}(k) \text { and } \hat{w}_{1}(k)=|k|^{-s} \hat{w}(k) .
$$

In particular, this implies

$$
\left\|u_{1}\right\|_{L^{2}}=\|u\|_{s},\left\|v_{1}\right\|_{L^{2}}=\|v\|_{s} \text { and }\left\|w_{1}\right\|_{L^{2}}=\|w\|_{-s} .
$$

We find then

$$
\begin{aligned}
\widehat{B(u, v)}(k) & =\frac{\alpha}{2} \frac{i k}{1+\beta|k|} \sum_{\substack{k_{1} \in \mathbb{Z}^{*} \\
k_{1} \neq k}} \frac{\hat{u}\left(k_{1}\right) \hat{v}\left(k-k_{1}\right)}{\sigma\left(k_{1}\right)+\sigma\left(k-k_{1}\right)-\sigma(k)} \\
& =\frac{\alpha}{2} \frac{i k}{1+\beta|k|} \sum_{\substack{k_{1} \in \mathbb{Z}^{*} \\
k_{1} \neq k}} \frac{|k|^{s} \hat{u}_{1}\left(k_{1}\right) \hat{v}_{1}\left(k-k_{1}\right)}{\left|k_{1}\right|^{s}\left|k-k_{1}\right|^{s}\left(\sigma\left(k_{1}\right)+\sigma\left(k-k_{1}\right)-\sigma(k)\right)} .
\end{aligned}
$$

Finally, it is enough to prove

$$
\left|\frac{\alpha}{2} \sum_{D} \frac{i k}{1+\beta|k|} \frac{|k|^{s} \hat{u}_{1}\left(k_{1}\right) \hat{v}_{1}\left(k-k_{1}\right) \hat{w}_{1}(k)}{\left|k_{1}\right|^{s}\left|k-k_{1}\right|^{s}\left(\sigma\left(k_{1}\right)+\sigma\left(k-k_{1}\right)-\sigma(k)\right)}\right| \leq C \frac{\alpha}{\beta^{2}}\left(\left\|u_{1}\right\|_{L^{2}}\left\|v_{1}\right\| L_{L^{2}}\right)\|w\|_{L^{2}} .
$$

\section{Lemma 2.3}

We have for $k$ and $k_{1}$ in $D$

$$
\left|\frac{i k}{1+\beta|k|} \frac{1}{\sigma\left(k_{1}\right)+\sigma\left(k-k_{1}\right)-\sigma(k)}\right| \leq \frac{4}{\beta^{2}} .
$$

Proof. According to the lemma 2.1, we have for $k$ and $k_{1}$ in $D$,

$$
\left|\frac{i k}{1+\beta|k|} \frac{1}{\sigma\left(k_{1}\right)+\sigma\left(k-k_{1}\right)-\sigma(k)}\right| \leq \frac{\left(1+\beta\left|k_{1}\right|\right)\left(1+\beta\left|k-k_{1}\right|\right)}{\beta^{2}\left|k_{1}\left(k-k_{1}\right)\right|} .
$$

Since $0<\beta \leq 1$ and $k, k_{1} \in \mathbb{Z}^{*}$, we have $1+\beta\left|k_{1}\right| \leq 2\left|k_{1}\right|$ and $1+\beta\left|k-k_{1}\right| \leq 2\left|k-k_{1}\right|$, thus

$$
\left|\frac{i k}{1+\beta|k|} \frac{1}{\sigma\left(k_{1}\right)+\sigma\left(k-k_{1}\right)-\sigma(k)}\right| \leq \frac{4}{\beta^{2}} .
$$

For $s \geq 0$, the triangle inequality implies

$$
\frac{|k|^{s}}{\left|k_{1}\right|^{s}\left|k-k_{1}\right|^{s}} \leq C\left(\frac{1}{\left|k_{1}\right|^{s}}+\frac{1}{\left|k-k_{1}\right|^{s}}\right),
$$

and thanks to the preceding lemma, it remains to bound

$$
\frac{\alpha}{\beta^{2}} \sum_{D} \frac{\left|\hat{u}_{1}\left(k_{1}\right)\right|\left|\hat{v}_{1}\left(k-k_{1}\right)\right|\left|\hat{w}_{1}(k)\right|}{\left|k_{1}\right|^{s}}+\frac{\alpha}{\beta^{2}} \sum_{D} \frac{\left|\hat{u}_{1}\left(k_{1}\right)\right|\left|\hat{v}_{1}\left(k-k_{1}\right)\right|\left|\hat{w}_{1}(k)\right|}{\left|k-k_{1}\right|^{s}}=: \text { I }+ \text { II. }
$$

The Cauchy-Schwarz inequality in $k$ gives

$$
\mathrm{I} \leq \frac{\alpha}{\beta^{2}}\left(\sum_{k \in \mathbb{Z}^{*}}\left(\sum_{\substack{k_{1} \in \mathbb{Z}^{*} \\ k_{1} \neq k}} \frac{\left|\hat{u}_{1}\left(k_{1}\right)\right|\left|\hat{v}_{1}\left(k-k_{1}\right)\right|}{\left|k_{1}\right|^{s}}\right)^{2}\right)^{1 / 2}\left(\sum_{k \in \mathbb{Z}^{*}}\left|\hat{w}_{1}(k)\right|^{2}\right)^{1 / 2}
$$


then the Cauchy-Schwarz inequality in $k_{1}$ is applied again,

$$
\mathrm{I} \leq \frac{\alpha}{\beta^{2}}\left(\sum_{\substack{k_{1} \in \mathbb{Z}^{*} \\ k_{1} \neq k}} \frac{1}{\left|k_{1}\right|^{2 s}}\right)^{1 / 2}\left(\sum_{k \in \mathbb{Z}^{*}} \sum_{\substack{k_{1} \in \mathbb{Z}^{*} \\ k_{1} \neq k}}\left|\hat{u}_{1}\left(k_{1}\right)\right|^{2}\left|\hat{v}_{1}\left(k-k_{1}\right)\right|^{2}\right)^{1 / 2}\left(\sum_{k \in \mathbb{Z}^{*}}\left|\hat{w}_{1}(k)\right|^{2}\right)^{1 / 2} .
$$

Since $s>1 / 2$, there exists $C>0$ such that

$$
\mathrm{I} \leq C \frac{\alpha}{\beta^{2}}\left(\left\|u_{1}\right\|_{L^{2}}\left\|v_{1}\right\|_{L^{2}}\right)\left\|w_{1}\right\|_{L^{2}} .
$$

By symmetry, we find similar inequality for II.

For $\delta>0$, we set $U_{\delta}:=\left\{u \in H_{0}^{s}(\mathbb{T}) ;\|u\|_{s}<\delta\right\}$. For $u \in H_{0}^{s}(\mathbb{T})$, we define the operator $\Lambda$ by,

$$
\Lambda u:=u+B(u, u) .
$$

The differential of this operator is given by, for all $\varphi \in \mathcal{C}_{\text {per }}^{\infty}(\mathbb{T})$

$$
\langle d \Lambda(u), \varphi\rangle=\varphi+2 B(u, \varphi)
$$

the preceding lemma implies that $d \Lambda$ is continuous from $H_{0}^{s}(\mathbb{T})$ to itself. Since $d \Lambda(0)$ is the identity, the inverse function theorem is applied to give the following lemma.

\section{Lemma 2.4}

Let $s>1 / 2$. Then there exist $0<\delta^{\prime}<\frac{\beta^{2}}{\alpha}, \delta>0$, and $C>0$ such that for all $v \in U_{\delta}$, there exists a unique $u \in U_{\delta^{\prime}}$ such that $\Lambda u=v$. Moreover

$$
\|u\|_{s} \leq C\|v\|_{s}
$$

and the map $\Lambda^{-1}$ is of class $\mathcal{C}^{1}$.

We aim to know which equation is satisfied by $\Lambda$, i.e. what is the normal form of the BO-BBM equation.

Lemma 2.5

Let $s>1 / 2$. There exists a trilinear operator

$$
F: H_{0}^{s}(\mathbb{T}) \times H_{0}^{s}(\mathbb{T}) \times H_{0}^{s}(\mathbb{T}) \longrightarrow H_{0}^{s}(\mathbb{T})
$$

satisfying that there exists a constant $C>0$ such that for all $\left(u_{1}, u_{2}, u_{3}\right) \in H_{0}^{s}(\mathbb{T}) \times H_{0}^{s}(\mathbb{T}) \times H_{0}^{s}(\mathbb{T})$

$$
\left\|F\left(u_{1}, u_{2}, u_{3}\right)\right\|_{s} \leq C \frac{\alpha^{2}}{\beta^{2}}\left\|u_{1}\right\|_{s}\left\|u_{2}\right\|_{s}\left\|u_{3}\right\|_{s}
$$

and, if $u \in \mathcal{C}\left([-T, T] ; H_{0}^{s}(\mathbb{T})\right)$ is solution of the equation (1.1), then $v$ defined by, for $t \in[-T, T]$

$$
v(t):=u(t)+B(u(t), u(t)),
$$

is solution of

$$
v_{t}+\frac{\partial_{x}}{1+\beta\left|\partial_{x}\right|} v=F(u, u, u)
$$


Proof. First of all we remark that $v \in \mathcal{C}\left([-T, T] ; H_{0}^{s}(\mathbb{T})\right)$ according to the lemma 2.2. We write

$$
\begin{aligned}
v_{t}+\frac{\partial_{x}}{1+\beta\left|\partial_{x}\right|} v & =u_{t}+\partial_{t} B(u, u)+\frac{\partial_{x}}{1+\beta\left|\partial_{x}\right|} u+\frac{\partial_{x}}{1+\beta\left|\partial_{x}\right|} B(u, u) \\
& =-\frac{\alpha}{2} \frac{\partial_{x}}{1+\beta\left|\partial_{x}\right|} u^{2}+\partial_{t} B(u, u)+\frac{\partial_{x}}{1+\beta\left|\partial_{x}\right|} B(u, u) .
\end{aligned}
$$

In one hand, we have

$$
\begin{aligned}
\partial_{t} B(u, u) & =B\left(u_{t}, u\right)+B\left(u, u_{t}\right)=2 B\left(u_{t}, u\right) \\
& =-\alpha \sum_{D} \mathrm{e}^{i k x} \frac{i k}{1+\beta|k|} \frac{\hat{u}_{t}\left(k_{1}\right) \hat{u}\left(k-k_{1}\right)}{\sigma\left(k_{1}\right)+\sigma\left(k-k_{1}\right)-\sigma(k)} .
\end{aligned}
$$

Since $u$ is solution of the equation (1.1), we obtain

$$
\hat{u}_{t}(k)=-\frac{\alpha}{2} \frac{i k}{1+\beta|k|} \widehat{u^{2}}(k)-\sigma(k) \hat{u}(k),
$$

thus, by symmetry, we have

$$
\begin{aligned}
\partial_{t} B(u, u)= & -\frac{\alpha^{2}}{2} \sum_{D} \mathrm{e}^{i k x} \frac{k k_{1}}{(1+\beta|k|)\left(1+\beta\left|k_{1}\right|\right)} \frac{\widehat{u^{2}}\left(k_{1}\right) \hat{u}\left(k-k_{1}\right)}{\sigma\left(k_{1}\right)+\sigma\left(k-k_{1}\right)-\sigma(k)} \\
& +\frac{\alpha}{2} \sum_{D} \mathrm{e}^{i k x} \frac{i k}{1+\beta|k|} \frac{\left(\sigma\left(k_{1}\right)+\sigma\left(k-k_{1}\right)\right) \hat{u}\left(k_{1}\right) \hat{u}\left(k-k_{1}\right)}{\sigma\left(k_{1}\right)+\sigma\left(k-k_{1}\right)-\sigma(k)} .
\end{aligned}
$$

In the other hand, we have

$$
\frac{\partial_{x}}{1+\beta\left|\partial_{x}\right|} B(u, u)=-\frac{\alpha}{2} \sum_{D} \mathrm{e}^{i k x} \frac{i k}{1+\beta|k|} \frac{\sigma(k) \hat{u}\left(k_{1}\right) \hat{u}\left(k-k_{1}\right)}{\sigma\left(k_{1}\right)+\sigma\left(k-k_{1}\right)-\sigma(k)} .
$$

The last term gives

$$
-\frac{\alpha}{2} \frac{\partial_{x}}{1+\beta\left|\partial_{x}\right|} u^{2}=-\frac{\alpha}{2} \sum_{D} \mathrm{e}^{i k x} \frac{i k}{1+\beta|k|} \hat{u}\left(k_{1}\right) \hat{u}\left(k-k_{1}\right) .
$$

Finally, we find

$$
v_{t}+\frac{\partial_{x}}{1+\beta\left|\partial_{x}\right|} v=-\frac{\alpha^{2}}{2} \sum_{D} \mathrm{e}^{i k x} \frac{k k_{1}}{(1+\beta|k|)\left(1+\beta\left|k_{1}\right|\right)} \frac{\widehat{u^{2}}\left(k_{1}\right) \hat{u}\left(k-k_{1}\right)}{\sigma\left(k_{1}\right)+\sigma\left(k-k_{1}\right)-\sigma(k)} .
$$

We consider then $D_{1}:=\left\{\left(k, k_{1}, k_{2}\right) \in \mathbb{Z}^{3} ; k \neq 0, k_{1} \neq 0, k_{2} \neq 0, k \neq k_{1}, k_{1} \neq k_{2}\right\}$ and we deduce from (2.2) that $F$ is defined by

$$
F\left(u_{1}, u_{2}, u_{3}\right):=-\frac{\alpha^{2}}{2} \sum_{D_{1}} \mathrm{e}^{i k x} \frac{k k_{1}}{(1+\beta|k|)\left(1+\beta\left|k_{1}\right|\right)} \frac{\hat{u}_{1}\left(k_{2}\right) \hat{u}_{2}\left(k_{1}-k_{2}\right) \hat{u}_{3}\left(k-k_{1}\right)}{\sigma\left(k_{1}\right)+\sigma\left(k-k_{1}\right)-\sigma(k)} .
$$

\section{Lemma 2.6}

We have for $k, k_{1}$ and $k_{2}$ in $D_{1}$

$$
\left|\frac{k k_{1}}{(1+\beta|k|)\left(1+\beta\left|k_{1}\right|\right)} \frac{1}{\sigma\left(k_{1}\right)+\sigma\left(k-k_{1}\right)-\sigma(k)}\right| \leq \frac{2}{\beta^{2}} .
$$


Proof. According to the lemma 2.1, we have for $k, k_{1}$ and $k_{2}$ in $D_{1}$,

$$
\left|\frac{k k_{1}}{(1+\beta|k|)\left(1+\beta\left|k_{1}\right|\right)} \frac{1}{\sigma\left(k_{1}\right)+\sigma\left(k-k_{1}\right)-\sigma(k)}\right| \leq \frac{1+\beta\left|k-k_{1}\right|}{\beta^{2}\left|k-k_{1}\right|},
$$

and since $0<\beta \leq 1$ and $k, k_{1} \in \mathbb{Z}^{*}$, we have $1+\beta\left|k-k_{1}\right| \leq 2\left|k-k_{1}\right|$, thus

$$
\left|\frac{k k_{1}}{(1+\beta|k|)\left(1+\beta\left|k_{1}\right|\right)} \frac{1}{\sigma\left(k_{1}\right)+\sigma\left(k-k_{1}\right)-\sigma(k)}\right| \leq \frac{2}{\beta^{2}} .
$$

In the same manner as lemma 2.2 , by duality, it is enough to bound

$$
\mathrm{I}:=\frac{\alpha^{2}}{2 \beta^{2}} \sum_{D_{1}} \frac{|k|^{s}\left|\hat{u}_{1}\left(k_{2}\right)\right|\left|\hat{u}_{2}\left(k_{1}-k_{2}\right)\right|\left|\hat{u}_{3}\left(k-k_{1}\right)\right|\left|\hat{u}_{4}(k)\right|}{\left|k_{2}\right|^{s}\left|k_{1}-k_{2}\right|^{s}\left|k-k_{1}\right|^{s}} .
$$

The Cauchy-Schwarz inequality is applied first in $k$ to give

$$
\mathrm{I} \leq \frac{\alpha^{2}}{\beta^{2}}\left(\sum_{k \in \mathbb{Z}^{*}}\left(\sum_{\substack{\left(k_{1}, k_{2}\right) \in\left(\mathbb{Z}^{*}\right)^{2} \\ k_{1} \neq k, k_{2} \neq k_{1}}} \frac{|k|^{s}\left|\hat{u}_{1}\left(k_{2}\right)\right|\left|\hat{u}_{2}\left(k_{1}-k_{2}\right)\right|\left|\hat{u}_{3}\left(k-k_{1}\right)\right|}{\left|k_{2}\right|^{s}\left|k_{1}-k_{2}\right|^{s}\left|k-k_{1}\right|^{s}}\right)^{2}\right)^{1 / 2}\left\|u_{4}\right\|_{L^{2}},
$$

and then in $\left(k_{2}, k_{1}\right)$,

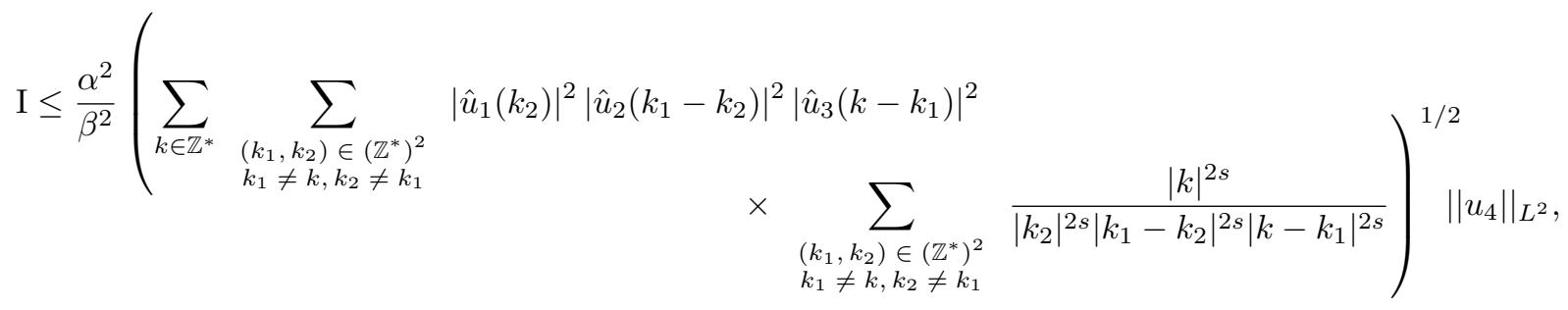

However, since $s>1 / 2 \geq 0$, the triangle inequality implies

$$
\frac{|k|^{2 s}}{\left|k_{2}\right|^{2 s}\left|k_{1}-k_{2}\right|^{2 s}\left|k-k_{1}\right|^{2 s}} \leq C\left(\frac{1}{\left|k_{2}\right|^{2 s}\left|k_{1}-k_{2}\right|^{2 s}}+\frac{1}{\left|k_{2}\right|^{2 s}\left|k-k_{1}\right|^{2 s}}+\frac{1}{\left|k_{1}-k_{2}\right|^{2 s}\left|k-k_{1}\right|^{2 s}}\right),
$$

thus

$$
\sup _{k \in \mathbb{Z}^{*}} \sum_{\substack{\left(k_{1}, k_{2}\right) \in\left(\mathbb{Z}^{*}\right)^{2} \\ k_{1} \neq k, k_{2} \neq k_{1}}} \frac{|k|^{2 s}}{\left|k_{2}\right|^{2 s}\left|k_{1}-k_{2}\right|^{2 s}\left|k-k_{1}\right|^{2 s}}<+\infty .
$$

We now prove the main result of this paper.

Proof of the theorem 0.2. We suppose $t>0$, the proof being similar for negative time. Let $\delta$ and $\delta^{\prime}$ the positive constants involved in the lemma 2.4.

According to the local well-posedness theorem 1.1, there exists $\varepsilon_{0}>0$ such that if $f \in U_{\varepsilon}$, for $\left.\varepsilon \in\right] 0, \varepsilon_{0}[$, then for $t \leq C_{2} \varepsilon^{-1} \frac{\beta}{\alpha}$,

$$
u(t)+B(u, u)(t) \in U_{\delta} \text { and } u(t) \in U_{\delta^{\prime}} .
$$


Thus $u(t)=\Lambda^{-1} v(t)$ for $t \leq C_{2} \varepsilon^{-1} \frac{\beta}{\alpha}=: T$.

The Duhamel formula gives for $t \in[0, T]$

$$
v(t)=S_{t}(f+B(f, f))+\int_{0}^{t} S_{t-\tau}(F(u, u, u))(\tau) d \tau,
$$

with $S_{t} f:=\sum_{k \in \mathbb{Z}^{*}} \mathrm{e}^{i k x} \mathrm{e}^{-i t \frac{k}{1+\beta|k|}} \hat{f}(k)$. Thanks to the lemma 2.2, there exists $C_{1}>0$ such that we have

$$
\begin{aligned}
\left\|S_{t}(f+B(f, f))\right\|_{s} & \leq\|f\|_{s}+\|B(f, f)\|_{s} \\
& \leq\|f\|_{s}+C \frac{\alpha}{\beta^{2}}\|f\|_{s}^{2} \leq C_{1} \varepsilon\left(1+\varepsilon \frac{\alpha}{\beta^{2}}\right) .
\end{aligned}
$$

Even if we take $\varepsilon_{0}>0$ smaller, we have $\varepsilon<\frac{\beta^{2}}{\alpha}$ and $1<\left(1+\varepsilon \frac{\alpha}{\beta^{2}}\right)<2$, and we deduce

$$
\left\|S_{t}(f+B(f, f))\right\|_{s} \leq 2 C_{1} \varepsilon .
$$

The lemmas 2.4 and 2.5 imply that there exists $C_{3}>0$ such that if $v(t) \in U_{\delta^{\prime}}$ for $t \in[0, T]$, then

$$
\left\|\int_{0}^{t} S_{t-\tau}\left(F\left(\Lambda^{-1} v, \Lambda^{-1} v, \Lambda^{-1} v\right)\right)(\tau) d \tau\right\|_{L^{\infty}\left([0, T] ; H_{0}^{s}(\mathbb{T})\right)}^{\leq C_{3}} \frac{\alpha^{2}}{\beta^{2}} T\|v\|_{L^{\infty}\left([0, T] ; H_{0}^{s}(\mathbb{T})\right)}^{3} .
$$

Even if we take $\varepsilon_{0}>0$ smaller, we impose $2 C_{1} \varepsilon_{0}<\delta$. We set $C_{0}=\frac{1}{54 C_{1}^{2} C_{3}}$ and $T_{0}=C_{0}\left(\varepsilon^{-1} \frac{\beta}{\alpha}\right)^{2}$. It follows then

$$
\|v\|_{L^{\infty}\left(\left[0, T_{0}\right] ; H_{0}^{s}(\mathbb{T})\right)} \leq 3 C_{1} \varepsilon .
$$

Let us suppose that the inequality (2.6) fails. Since

$$
\|v(0)\|_{s}=\|f+B(f, f)\|_{s} \leq C_{1} \varepsilon\left(1+\varepsilon \frac{\alpha}{\beta^{2}}\right)<3 C_{1} \varepsilon,
$$

by continuity with time, there exists $\tau \in\left[0, T_{0}\right]$ such that for $t \in[0, \tau]$

$$
\|v(t)\|_{s} \leq 3 C_{1} \varepsilon \text { and }\|v(\tau)\|_{s}=3 C_{1} \varepsilon .
$$

Let $C$ the positive constant involved in the lemma 2.4, we also impose $3 C_{1} C \varepsilon_{0}<\delta^{\prime}$. We know that $u(t) \in U_{\delta^{\prime}}$ for $|t| \leq T$, and with this choice of $\varepsilon$, it follows that $u(t) \in U_{\delta^{\prime}}$ for $t \in[0, \tau]$. Indeed, if there exists $\tau_{1} \in[0, \tau]$ such that for $t \in\left[0, \tau_{1}\right]$

$$
\|u(t)\|_{s}<\delta^{\prime} \text { and }\left\|u\left(\tau_{1}\right)\right\|_{s}=\delta^{\prime},
$$

then by continuity with time and according to the lemma 2.4 , we have

$$
\begin{aligned}
\delta^{\prime}=\left\|u\left(\tau_{1}\right)\right\|_{s} & =\lim _{t \rightarrow \tau_{1}}\|u(t)\|_{s} \leq \sup _{0 \leq t<\tau_{1}}\|u(t)\|_{s} \\
& \leq \sup _{0 \leq t<\tau_{1}} C\|v(t)\|_{s} \leq 3 C_{1} C \varepsilon<\delta^{\prime},
\end{aligned}
$$

which is a contradiction.

Finally, we find from the Duhamel formula (2.3) and inequalities (2.4) and (2.5)

$$
\|v\|_{L^{\infty}\left([0, \tau] ; H_{0}^{s}(\mathbb{T})\right)} \leq 2 C_{1} \varepsilon+C_{3} \frac{\alpha^{2}}{\beta^{2}} \tau\|v\|_{L^{\infty}\left([0, \tau] ; H_{0}^{s}(\mathbb{T})\right)}^{3},
$$


or equivalently

$$
3 C_{1} \varepsilon \leq 2 C_{1} \varepsilon+C_{3} \frac{\alpha^{2}}{\beta^{2}} \tau\left(3 C_{1} \varepsilon\right)^{3},
$$

which gives

$$
\tau \geq 2 C_{0}\left(\varepsilon^{-1} \frac{\beta}{\alpha}\right)^{2}=2 T_{0} .
$$

This is a contradiction with the fact $\tau \in\left[0, T_{0}\right]$. Then the inequality (2.6) is true and using the lemma 2.4 , it get for $t \in\left[0, T_{0}\right]$

$$
\|u(t)\|_{s} \leq C\|v(t)\|_{s} \leq 3 C_{1} C \varepsilon
$$

Remark 2 Nothing says, in this proof, that the bound in time $\left(\varepsilon^{-1} \frac{\beta}{\alpha}\right)^{2}$ is optimal.

Remark 3 In the continuous case $(x \in \mathbb{R})$, we can use the dispersive properties of the linear evolution via the Van der Corput's lemma, these properties being false in the periodic case. More precisely, we already proved [11] that, for a initial datum of size $\varepsilon$, for $2<q<+\infty$, there exists $C>0$ such that for all $t \in \mathbb{R}$

$$
\|u(t)\|_{L^{q}} \leq C \varepsilon .
$$

The proof requires to impose a nonlinearity of type $u^{\rho} u_{x}$ with $\rho \geq 3$.

\section{Remarks about the KP equations}

In this section, we slightly improve the result found by Tzvetkov [15] by considering the KP-II equation rewritten under the form

$$
u_{t}+u_{x}+\alpha u u_{x}+\beta u_{x x x}+\gamma \partial_{x}^{-1} u_{y y}=0 .
$$

Here, $\partial_{x}^{-1}$ denotes the anti-derivative, defined such that $\widehat{\partial_{x}^{-1} u}(k):=\frac{\widehat{u}(k)}{i k}, \alpha$ the quotient between the characteristic waves amplitude and the depth of the fluid, $\beta$ the square of the quotient between this depth and the wavelength in the $x$-direction and $\gamma$ the square of the quotient between the wavelengths in the two directions of the surface. This equation results from the modelling of the small amplitude long waves in shallow water moving mainly in the $x$-direction. In concrete terms, if we consider such waves propagating on the Pacific ocean, i.e. for an average depth of 4000 metres, the studied waves have an amplitude around $1 \mathrm{~m}$, for a wavelength $213 \mathrm{~km}$ [6], in particular $\alpha=\frac{1}{4000}=2.5 \times 10^{-4}$ and $\beta=\left(\frac{4000}{213000}\right)^{2} \simeq 3.5 \times 10^{-4}$.

The global in time well-posedness of the KP-II equation is proved by Bourgain [4] for all initial data $f \in H_{0}^{s}\left(\mathbb{T}^{2}\right)$, with $s \geq 0$. In particular, the mass

$$
\|u(t)\|_{L^{2}}=\|f\|_{L^{2}}
$$

is conserved for all time $t \in \mathbb{R}$. The local in time well-posedness [9] implies that, for $s>2$, there exist $C_{1}>0$ and $C_{2}>0$, depending only on $s$, such that, if $\|f\|_{s} \leq \varepsilon$, then for $|t| \leq C_{2} \varepsilon^{-1} \frac{1}{\alpha}$,

$$
\|u(t)\|_{s} \leq C_{1} \varepsilon
$$


The dispersive relation $\sigma$, defined for $(k, l) \in \mathbb{Z}^{2}$, by $\sigma(k, l):=i\left(k-\beta k^{3}+\gamma \frac{l^{2}}{k}\right)$, verifies for $(k, l)$ and $\left(k_{1}, l_{1}\right)$ in $\mathbb{Z}^{2}$, with $k \neq 0, k_{1} \neq 0$ and $k \neq k_{1}$,

$$
\left|\sigma\left(k_{1}, l_{1}\right)+\sigma\left(k-k_{1}, l-l_{1}\right)-\sigma(k, l)\right| \geq 3 \beta\left|k k_{1}\left(k-k_{1}\right)\right| .
$$

Let $D:=\left\{\left(k, l, k_{1}, l_{1}\right) \in \mathbb{Z}^{4} ; k \neq 0, k_{1} \neq 0, k \neq k_{1}\right\}$, we define the bilinear operator

$$
B(u, v):=-\frac{\alpha}{2} \sum_{D} \mathrm{e}^{i k x+i l y} \frac{i k \hat{u}\left(k_{1}, l_{1}\right) \hat{v}\left(k-k_{1}, l-l_{1}\right)}{\sigma\left(k_{1}, l_{1}\right)+\sigma\left(k-k_{1}, l-l_{1}\right)-\sigma(k, l)} .
$$

We can prove similar lemmas as 2.2, 2.4 and 2.5.

\section{Lemma 3.1}

Let $s>1$. There exists $C>0$ such that for all $u$ and $v$ in $H_{0}^{s}\left(\mathbb{T}^{2}\right)$

$$
\|B(u, v)\|_{s} \leq C \frac{\alpha}{\beta}\|u\|_{s}\|v\|_{s} .
$$

There exists a trilinear operator

$$
F: H_{0}^{s}\left(\mathbb{T}^{2}\right) \times H_{0}^{s}\left(\mathbb{T}^{2}\right) \times H_{0}^{s}\left(\mathbb{T}^{2}\right) \longrightarrow H_{0}^{s}\left(\mathbb{T}^{2}\right)
$$

satisfying that there exists $C>0$ such that for all $\left(u_{1}, u_{2}, u_{3}\right) \in H_{0}^{s}\left(\mathbb{T}^{2}\right) \times H_{0}^{s}\left(\mathbb{T}^{2}\right) \times H_{0}^{s}\left(\mathbb{T}^{2}\right)$

$$
\left\|F\left(u_{1}, u_{2}, u_{3}\right)\right\|_{s} \leq C \frac{\alpha^{2}}{\beta}\left\|u_{1}\right\|_{s}\left\|u_{2}\right\|_{s}\left\|u_{3}\right\|_{s}
$$

Moreover, if $u \in \mathcal{C}\left([-T, T] ; H_{0}^{s}\left(\mathbb{T}^{2}\right)\right)$ is solution of the equation of the KP-II equation, then $v$ defined by, for $t \in[-T, T]$

$$
v(t):=u(t)+B(u(t), u(t))
$$

is solution of

$$
v_{t}+v_{x}+\beta v_{x x x}+\gamma \partial_{x}^{-1} v_{y y}=F(u, u, u) .
$$

In the same way as for the theorem 0.2 , for $s>1$, there exist $0<\varepsilon_{0}<\frac{\beta}{\alpha}$ and $C_{1}>0$ such that if $\|f\|_{s} \leq \varepsilon$, the bound (3.1) can be extended until the time

$$
T=C_{1}\left(\varepsilon^{-1} \frac{\sqrt{\beta}}{\alpha}\right)^{2}
$$

Here again, the constraint of smallness on $\varepsilon_{0}$ does not depend on $\alpha$ and $\beta$ when $\frac{\beta}{\alpha} \geq 1$, and the fact that $\varepsilon_{0}$ is chosen in a such way $\varepsilon<\frac{\beta}{\alpha}$, with $0<\varepsilon<\varepsilon_{0}$, implies that $\left(\varepsilon^{-1} \frac{\sqrt{\beta}}{\alpha}\right)^{2} \geq \varepsilon^{-1} \frac{1}{\alpha}$. If $\alpha=\beta=\gamma=1$, we find the result proved by Tzvetkov [15] again. We notice that the transverse $y$-direction, given by $\gamma$, has no effect on the bound in time.

Let us consider now the KP-BBM-II equation (regularized version of KP-II)

$$
v_{t}+v_{x}+\alpha v v_{x}-\beta v_{x x t}+\gamma \partial_{x}^{-1} v_{y y}=0 .
$$


We can also prove the global well-posedness of the Cauchy problem [14] and the norm $\|u(t)\|_{1}$ is conserved for all time $t \in \mathbb{R}$. As a consequence of the local in time well-posedness of the Cauchy problem, for $s>1$, there exist $C_{1}>0$ and $C_{2}>0$ such that if $\|f\|_{s} \leq \varepsilon$, then for $|t| \leq C_{2} \varepsilon^{-1} \frac{\beta}{\alpha}$, the solution $v$ satisfies

$$
\|v(t)\|_{s} \leq C_{1} \varepsilon .
$$

But this bound (3.2) can not be extended more long time by using the normal form method. Indeed, if we denote for $(k, l) \in \mathbb{Z}^{2}, \sigma(k, l):=i \frac{k+\gamma \frac{l^{2}}{k}}{1+\beta k^{2}}$, then for $(k, l)$ and $\left(k_{1}, l_{1}\right)$ in $\mathbb{Z}^{2}$, with $k \neq 0, k_{1} \neq 0$ and $k \neq k_{1}$, the dispersive relation $\sigma$ verifies

$$
\begin{aligned}
\left|\sigma\left(k_{1}, l_{1}\right)+\sigma\left(k-k_{1}, l-l_{1}\right)-\sigma(k, l)\right|=\mid \beta(3+\beta & \left.\left(k_{1}^{2}-k k_{1}+k^{2}\right)\right)+R \mid \\
& \times \frac{\left|k k_{1}\left(k-k_{1}\right)\right|}{\left(1+\beta k^{2}\right)\left(1+\beta k_{1}^{2}\right)\left(1+\beta\left(k-k_{1}\right)^{2}\right)},
\end{aligned}
$$

where $R=R\left(k, k_{1}, l, l_{1}, \gamma, \beta\right)$ can be negative, then the equality (3.3) can vanish. The first term in (3.3) can be used to find similar long time bounds for the BBM equation.

\section{References}

[1] V. I. Arnold, Geometric Methods in the Theory of Ordinary Differential Equations, Springer-Verlag, New York, 1983.

[2] T. B. Benjamin, Internal waves of permanent form in fluids of great depth, J. Fluid. Mech. 29 (1967) 559-592.

[3] T. B. Benjamin, J. L. Bona, J. J. Mahony, Model equations for long waves in nonlinear dispersive systems, Philos. Trans. Roy. Soc. London Ser. A 272 (1220) (1972) 47-78.

[4] J. Bourgain, On the Cauchy problem for the Kadomtsev-Petviashvili equation, Geom. Funct. Anal. 3 (4) (1993) 315-341.

[5] H. Brézis, T. Gallouët, Nonlinear Schrödinger evolution equations, Nonlinear Anal. 4 (4) (1980) 677-681.

[6] International Tsunami Information Centre, Tsunami: les grandes vagues, Brochure du programme Tsunami de l'UNESCO, 2003.

[7] R. Davis, A. Acrivos, Solitary internal waves in deep water, J. Fluid. Mech. 29 (1967) 593-608.

[8] R. J. Iório, Jr., KdV, BO and friends in weighted Sobolev spaces, in: Functional-analytic methods for partial differential equations (Tokyo, 1989), vol. 1450 of Lecture Notes in Math., Springer, Berlin, 1990, pp. 104-121.

[9] R. J. Iório, Jr., W. V. L. Nunes, On equations of KP-type, Proc. Roy. Soc. Edinburgh Sect. A 128 (4) (1998) $725-743$.

[10] C. R. Jackson, An Atlas of Internal Solitary-like Waves and their Properties, Global ocean associates, 2004.

[11] Y. Mammeri, On the decay in time of solutions of some generalized regularized long waves equations, Commun. Pure Appl. Anal. 7 (2008), no. 3, 513-532.A

[12] Y. Matsuno, A unified theory of nonlinear wave propagation in two-layer fluid systems, J. Phys. Soc. Jpn. 62 (6) (1993) 1902-1916.

[13] H. Ono, Algebraic solitary waves in stratified fluids, J. Phys. Soc. Japan 39 (4) (1975) 1082-1091.

[14] J.-C. Saut, N. Tzvetkov, Global well-posedness for the KP-BBM equations, AMRX Appl. Math. Res. Express (1) (2004) 1-16.

[15] N. Tzvetkov, Long time bounds for the periodic KP-II equation, Int. Math. Res. Not. (46) (2004) 2485-2496. 